\title{
Accuracy Analysis and Motion Control of Two-axis Nonmagnetic Turntable Based on Ultrasonic Motor
}

\author{
Zhuo WANG*, Xin-tong WANG*, Tao WANG**, Bo ZHANG*, Hong-wen MA* \\ *College of Mechanical and Electrical Engineering, Harbin Engineering University, Harbin 150001, PR China, \\ E-mail:2958219945@qq.com \\ **School of Mechanical Engineering, Hebei University of Technology, Tianjin, 300401, PR China

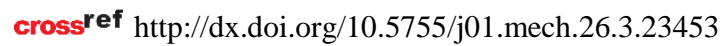

\section{Introduction}

The turntable is generally divided into an inertial test bench and a simulation turntable for calibration, performance testing, and semi-physical simulation of aircraft guidance products $[1,2]$, testing the attitude angle variation of a single carrier or the relative motion between multiple carriers, the docking process, and other parameters $[3,4]$.

In 1954, the turret developed by the Massachusetts Institute of Technology used photoelectric angle-measuring sensors and precision bearings, which improved the precision greatly and hence was put into production $[5,6]$. At present, the domestic and international turntables have high precision and fast response, providing high calibration accuracy and accurate performance detection for the calibration and testing of inertial navigation components [7, 8]. With the advancement of industrial control technology, the turntable has been rapidly developed [9, 10]. In 2013, the five-axis flight simulation turret developed by Acutronic has successfully been able to meet the high-precision requirements for testing high-dynamic missile performance.

The turntable is a high-precision turntable for seeker testing. The rotary table is driven by an ultrasonic motor to ensure the nonmagnetic and high-precision requirements of the turntable $[11,12]$. Ultrasonic motors use the linear effects of piezoelectric materials to achieve linear or rotational motion through frictional coupling. They do not distort the magnetic field and achieve high precision [13, 14]. The turntable uses ceramic bearings to ensure rotational accuracy. The support method adopts an adjustable threelegged positioning. A level is installed on the bottom plate to observe whether the turntable is installed horizontally. The turntable is equipped with a very high precision grating encoder to ensure that the measurement accuracy is higher than the control accuracy.

In this study, the pointing accuracy of the turntable was studied. The influence of the grating installation error on the accuracy detection was also analyzed. The steadystate error of the turntable has an important influence on the motion accuracy of the turntable. Finally, the influence of the control parameters on the steady-state error of the turntable was analyzed. The positioning accuracy and control parameters of the two-axis precision nonmagnetic turntable were obtained, and the PID adjustment was introduced to make the accuracy index of the nonmagnetic turntable meet the requirements. The turntable could realize a nonmagnetic working environment and achieve the high precision required by the index under the driving of the ultrasonic motor [15].

\section{Two-axis precision turntable control system construction and index requirements}

\subsection{Control system construction}

Fig. 1 shows that the turntable has two modes: one is an automatic mode controlled by an industrial computer and the other is a manual mode controlled by a pulse handle. After the controller receives the signal, the HF2 and HF4 ultrasonic motors are driven by the driver so as to drive the azimuth axis and the pitch axis of the turntable, respectively [16]. During the rotation process, the RL encoder returns the current turntable position angle to the controller in real time, and the industrial computer and the controller are in twoway communication so that the current position is seen on the display of the industrial computer. These two modes are used to control the rotation of the turntable, observe the motion curve and the motion parameters through the industrial computer, and adjust the controller parameters so that the motion parameters meet the requirements of the index [17]. This system is used for specific experimental studies described in Sections 3.5 and 4.

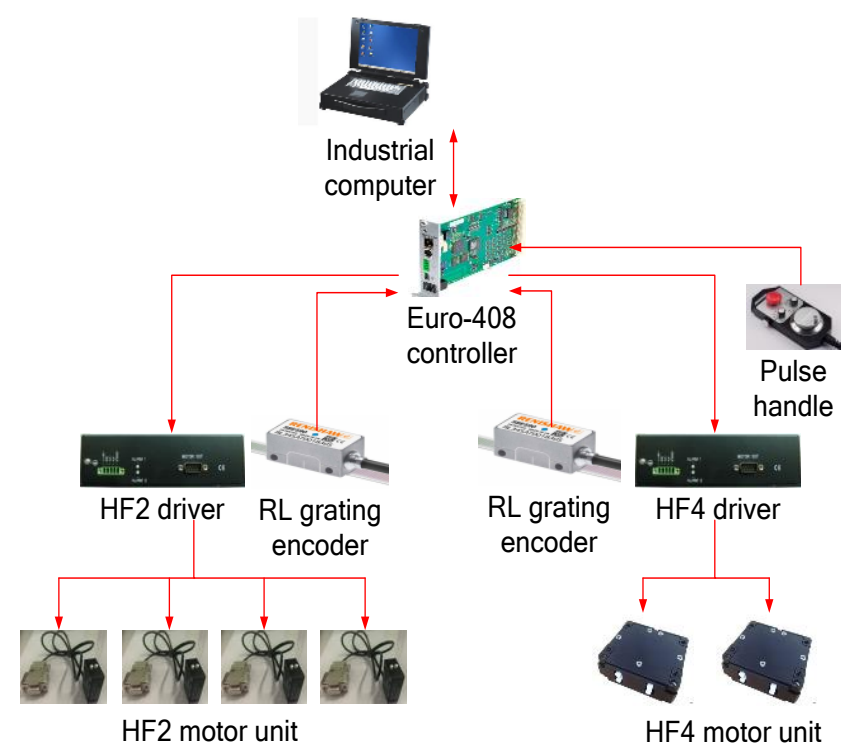

Fig. 1 Control system construction

2.2. Turntable indicator requirements

According to the positional search for the relative positional relationship of the infrared rays and the requirements of the performance parameters of the tester, the specific parameters of the two-axis precision nonmagnetic turntable are as follows: 
1. Azimuth-axis response time: $8 \mathrm{~ms}$.

2. Azimuth-axis steady-state error: $0.002^{\prime}$.

3. Azimuth adjustment time: $2.5 \mathrm{~s}$.

4. Pitch-axis steady-state error: $0.002^{\prime}$.

5. Pitch-axis adjustment time: $2.5 \mathrm{~s}$.

The response time, adjustment time, and steady-state error indicators of the two axes of the turntable are analyzed in Sections 3.4 and 4.2.

\section{Experimental analysis and influencing factors of accuracy of two-axis precision turntable}

\subsection{Factors affecting the accuracy of two-axis precision turntable}

In the calibration process of the positioner, only the rotation accuracy of the test turret is higher than the accuracy of the positioner seeker, and the test and calibration of the positioner can be realized. The black body radiation source is also used to simulate the target information to test the tracking function of a certain type of target. At this time, the pointing accuracy of the two-axis rotary table is critical for the calibration of the positioner.

The precision turntable is used for position tracking measurements of the front seeker of a certain type of missile. In the measurement, the blackbody radiation source emits infrared rays, and the infrared rays are reflected by the plane mirror to reach the seeker. The angle of the infrared mirror can be changed by changing the angle of the mirror. At this time, the turntable can make the seeker accurately track the infrared rays.

The pointing accuracy is the deviation of the actual spatial orientation of a device fixed on the turntable from the ideal spatial orientation. The test process has strict requirements for the precise positioning of the turntable. The precise positioning of the direct drive of the ultrasonic motor and the nanometer resolution of the encoder can realize the precise positioning of the two-axis turntable. The factors affecting the pointing accuracy of the two-axis turntable are mainly as follows:

1. The geometric error mainly includes dimensional error, shape and positional error, and accumulation error during the assembly process [18].

2 . The motion error mainly includes the motion rotation accuracy of each degree of freedom bearing and guide rail, the positioning error of the ultrasonic motor, the measurement error of the measuring component, and the zero error.

3. The control system error includes the stability of the control system, which affects the steady-state error of the turntable, and the nonlinearity of the ultrasonic motor, which is very strong. The requirements for the control system are high, and the targeted algorithm optimization for the ultrasonic motor is needed.

\subsection{Effect of grating encoder on accuracy}

Since the rotation axis of the grating disk and the rotation axis of the azimuth axis do not overlap absolutely, the azimuth axis has a circular runout during the movement, so the error accumulates as follows: the grating head has a large circular runout error with respect to the grating ruler. The grating measurement error is shown in Fig. 2. This section will analyze the effect of the grating eccentricity e on the measurement error. The resolution of the selected grating encoder in this subject is $50 \mathrm{~nm}$, and the mounting radius of the grating is $125 \mathrm{~mm}$, so that the angular resolution of the grating encoder is $1.44 \times 10^{-3 \prime \prime}$.The ideal resolution for turntable position detection is high.

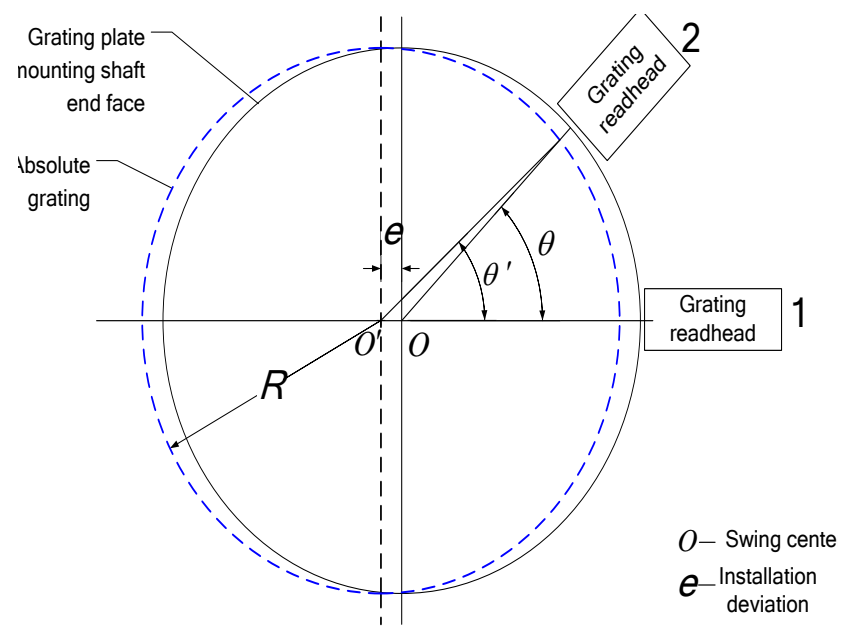

Fig. 2 Raster mounting eccentricity diagram

When the azimuth axis was rotated clockwise by $\theta$ $\left(0<\theta<90^{\circ}\right)$, the angle of the actual rotation of the grating was $\theta^{\prime}$, due to the existence of the grating eccentricity $e$; the corresponding angle $\Delta=\theta-\theta^{\prime}$ was the measurement error of the grating. Position 1 was defined as the zero point of the grating read head, and position 2 as the end position of the grating head; then, according to the angle relationship shown in Fig. 2:

$$
\frac{R}{\sin \theta}=\frac{e}{\sin \left(\theta-\theta^{\prime}\right)},
$$

derived:

$$
\Delta=\arcsin \left(\frac{e s i n \theta}{R}\right)
$$

Several cases, such as $90^{\circ}<\theta<180^{\circ}, 180^{\circ}<\theta<$ $270^{\circ}$, and $270^{\circ}<\theta<360^{\circ}$, were calculated, and the measurement error expression of the grating was obtained, as shown in Eq. (2). When $\theta=180^{\circ}$ and $\theta=360^{\circ}$, the grating measurement error $\Delta=0$ was obviously in line with the actual situation. The grating measurement error $\Delta$ was analyzed and calculated, and the relationship between the measurement error $\Delta$, grating rotation angle $\theta$, and the eccentricity $e$ was obtained, as shown in Fig. 3 .

The absolute value of the maximum measurement error was linear with the eccentricity $e$, and the variation in the grating measurement error was periodic. When the eccentricity $e=1 \mathrm{~mm}$, the theoretical measurement error of the grating was $\Delta=0.46^{\circ}$, and the measurement error was large. When the eccentricity was $0.1 \mathrm{~mm}$, the theoretical measurement error of the grating was $\Delta=0.046^{\circ}$. Therefore, to achieve a measurement error of $0.01^{\prime}$, the eccentricity $e$ of the grating measuring system should be guaranteed to be $0<e \leq 20.875 \mu \mathrm{m}$. By measuring the actual circular run out of the azimuth axis of the mechanical body of the turntable, the circular runout of the grating-mounted cylindrical surface was $0.03 \mathrm{~mm}$, that is, the eccentricity $e=0.015 \mathrm{~mm}$. 


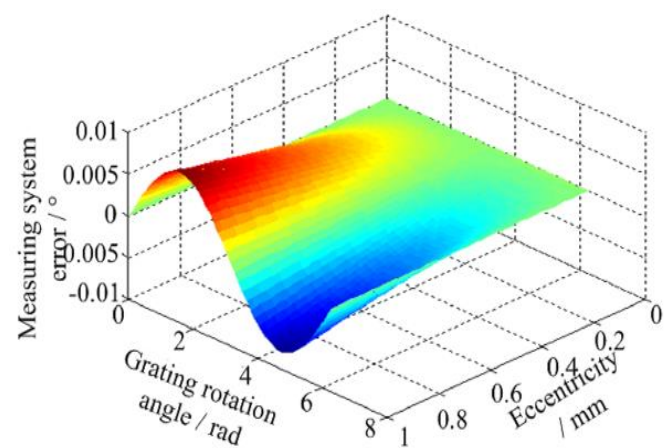

Fig. 3 Grating measurement error diagram

According to Eq. (2), the actual measurement error was $\Delta=\left(6.88 \times 10^{-3}\right)^{\circ}$, which was obviously greater than the technical requirement of $0.01^{\prime}$. The method for reducing the error of the turntable grating measuring system was to pre-install the azimuth axis on the bearing seat, correctly connect the grating mounting plate with the azimuth axis, and use the bearing seat as a processing reference to perform circular beating on the cylindrical surface of the grating ruler.

3.3. Influence of mechanical body of two-axis precision turntable on precision

In order to express the structural characteristics of the high-precision two-axis electric turntable concisely and clearly, a mathematical model of the pointing error of the two-axis precision turntable is established. The spatial coordinate system is established to simplify the abstraction of the two-axis precision turntable. Establish the right-hand Cartesian coordinate system of the initial position two-axis turntable. The $\mathrm{Y}$-axis and the pitch axis are collinear. The $\mathrm{X}$-axis is perpendicular to the paper plane and perpendicular to the $\mathrm{Y}$-axis. The $\mathrm{Z}$-axis is perpendicular to the plane formed by the $\mathrm{X}$ and $\mathrm{Y}$ axes and perpendicular to the turntable mounting base surface. The two-axis precision turret rotates from the starting position, and the azimuth axis and the pitch axis rotate $\theta_{1}, \theta_{2}$ angles around $\mathrm{Z}$ and $\mathrm{Y}$, respectively. The two-axis turntable has static error and motion error simultaneously during the movement, and the motion error changes continuously with the rotational angular velocity and the rotational position. The mathematical model involves many parameters, so only the static error of the two-axis turntable is modeled and analyzed [19]. The simplified two-axis turntable structure and error source are shown in Fig. 4.

In an ideal state, the positioner was installed at a position on the T-shaped groove table of the two-axis turntable. After the two degrees of freedom of the two-axis turntable were rotated through a certain angle, the pointing vector $P$ of the positioner was converted into $P_{1}$, a Euler transform in an ideal state. The matrix was $R_{1}$, and the relationship between them was:

$$
\vec{P}_{1}=R_{1} \vec{P}
$$

The Cardan angle was rotated between adjacent coordinate systems. The coordinate system $\{A\}$ coincided with the origin of $\{B\}$, and $\{B\}$ was rotated by $\theta_{1}$ angle around the $X_{A}$ axis of $\{A\}$ and around the $\{A\}$ in the new state. The $Y_{A}$ axis was rotated by $\theta_{2}$, and in the new state, it was rotated by $\theta_{3}$ around the $Z_{A}$ axis of $\{A\}$ (the rotation angle was small). If the $X_{B}, Y_{B}$, and $Z_{B}$ axes of the $\{B\}$ coordinate system had the translational quantities $\delta_{1}, \delta_{2}$, and $\delta_{3}$ with respect to the $X_{A}, Y_{A}$, and $Z_{A}$ axes of $\{A\}$, respectively, the homogeneous transformation matrix between the two coordinate systems was written as follows:

$$
{ }_{B}^{A} T=\left[\begin{array}{cc}
{ }_{B}^{A} R & { }^{A} P_{B_{O}} \\
0 & 1
\end{array}\right] \approx\left[\begin{array}{llll}
1 & -\theta_{3} & \theta_{2} & \delta_{1} \\
\theta_{3} & 1 & -\theta_{1} & \delta_{2} \\
-\theta_{2} & \theta_{1} & 1 & \delta_{3} \\
0 & 0 & 0 & 1
\end{array}\right],
$$

where: ${ }_{B}^{A} R$ is a $3 \times 3$ matrix describing the orientation of $\{B\}$ relative to $\{A\} ;{ }^{A} P_{B_{O}}$ is a $3 \times 1$ matrix describing the position of the coordinate origin of $\{B\}$ relative to $\{A\}$.

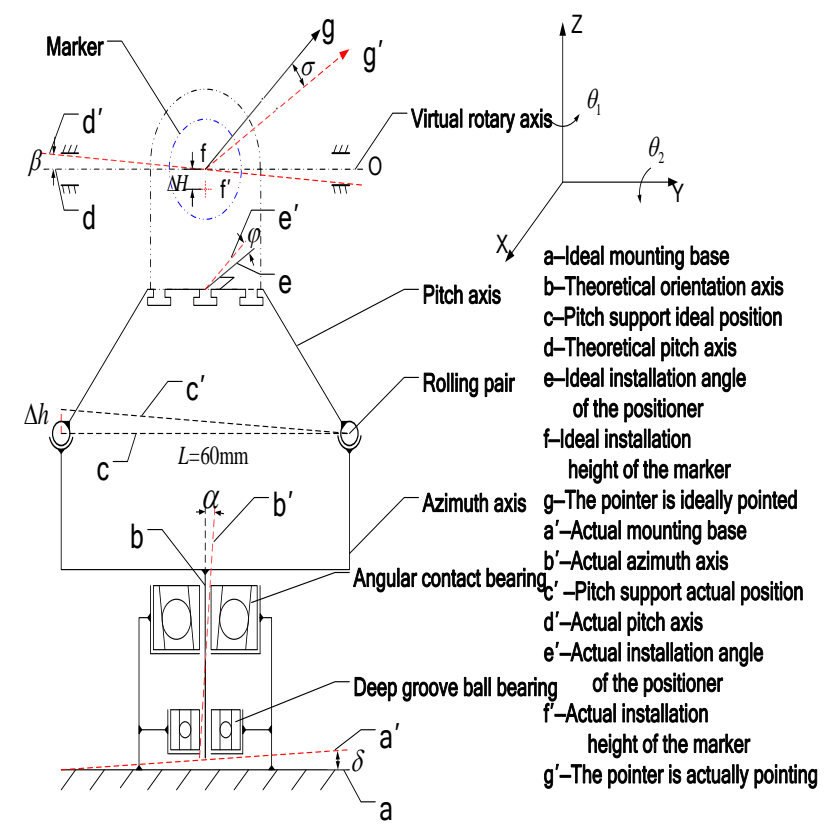

Fig. 4 Source analysis of the two-axis turntable error

For any point ${ }^{B} P$ in $\{B\}$, it was described in $\{A\}$ as:

$$
\left[\begin{array}{c}
{ }^{A} p \\
1
\end{array}\right]=\left[\begin{array}{cc}
{ }_{B}^{A} R & { }^{A} P_{B_{O}} \\
0 & 1
\end{array}\right]\left[\begin{array}{c}
{ }^{B} p \\
1
\end{array}\right] .
$$

${ }_{B}^{A} T$ is a square coordinate transformation square matrix of size $4 \times 4$, which comprehensively exhibits translational transformation and rotational transformation of coordinates. The transformation matrix of the positioner under ideal conditions was obtained by the following homogeneous transformation method:

$$
R_{1}=\left[\begin{array}{cccc}
\cos \theta_{1} & \sin \theta_{1} & 0 & 0 \\
-\sin \theta_{1} & \cos \theta_{1} & 0 & 0 \\
0 & 0 & 1 & 0 \\
0 & 0 & 0 & 1
\end{array}\right] \cdot\left[\begin{array}{cccc}
\cos \theta_{2} & 0 & -\sin \theta_{2} & 0 \\
0 & 1 & 0 & 0 \\
\sin \theta_{2} & 0 & \cos \theta_{2} & 0 \\
0 & 0 & 0 & 1
\end{array}\right] .(6)
$$

After the positioner was installed in the T-shaped slot table, the ideal pointing vector of the positioner was 
$\vec{P}_{1}=\left[\begin{array}{lll}1 & 0 & 0\end{array}\right]^{T}$, when the axes of the turntable were in the initial position without introducing various errors. After the two-axis turntable was rotated by two axes, in the ideal case, the initial vector was transformed into a new pointing vector $P_{1}$. Substituting Eq. (6) into Eq. (3):

$$
\begin{aligned}
& {\left[\begin{array}{c}
P_{1} \\
1
\end{array}\right]=\left[\begin{array}{cccc}
\cos \theta_{1} & \sin \theta_{1} & 0 & 0 \\
-\sin \theta_{1} & \cos \theta_{1} & 0 & 0 \\
0 & 0 & 1 & 0 \\
0 & 0 & 0 & 1
\end{array}\right] .} \\
& {\left[\begin{array}{cccc}
\cos \theta_{2} & 0 & -\sin \theta_{2} & 0 \\
0 & 1 & 0 & 0 \\
\sin \theta_{2} & 0 & \cos \theta_{2} & 0 \\
0 & 0 & 0 & 1
\end{array}\right]\left[\begin{array}{c}
P \\
1
\end{array}\right] .}
\end{aligned}
$$

In the actual process, the pointing accuracy of the two-axis turntable is affected by various factors, and the error introduced by the processing of the turntable parts as well as the turntable assembly cannot be ignored. The initial pointing of the positioner cannot go to $P$ from $P_{1}$ and actually points to $P_{2}$, and is given by:

$$
\vec{P}_{2}=R_{2} \vec{P}
$$

where: $R_{2}$ is a transformation matrix with errors; $\vec{P}_{2}$ is the actual pointing vector.

In the actual situation, the factors affecting the twoaxis turntable are the turret mounting base error $\delta$, the azimuth-axis error $\alpha$, the positional accuracy of the azimuth axis and the pitch axis $\Delta \theta_{1}$ and $\Delta \theta_{2}$, the pitch-axis error $\beta$ caused by the rolling pair height error $\Delta h$, the perpendicularity $p$ of the rotary axis, the mounting angle error $\varphi$ of the positioner mounting base, and the mounting height error of the positioner. The transformation matrix of the two-axis turntable with errors was as follows:

$$
R_{2}=A_{\delta} \cdot O_{\alpha} \cdot I_{\beta} \cdot W_{(\varphi, \Delta H)},
$$

where: $A_{\delta}$ is the mounting base error transfer matrix; $O_{\alpha}$ is the Azimuth-axis error transfer matrix; $I_{\beta}$ is the pitch-axis error transfer matrix; $W_{(\varphi, \Delta h)}$ is the positioner installation error transfer matrix.

Substituting Eq. (9) and the initial pointing vector into Eq. (8) yielded the actual pointing vector:

$$
\left[\begin{array}{ll}
P_{2} & 1
\end{array}\right]^{T}=A_{\delta} \cdot O_{\alpha} \cdot I_{\beta} \cdot W_{(\varphi, \Delta H)} \cdot\left[\begin{array}{ll}
P & 1
\end{array}\right]^{T} .
$$

First, the influence of the installation error of the positioner on the pointing error was analyzed. The actual installation height of the positioner and the theoretical installation centerline were highly deviated. A deviation $\varphi$ existed between the axis of the positioner and the verticality of the rotary axis. The error was introduced according to the spatial coordinate transformation relationship matrix $W_{(\varphi, \Delta h)}$ :

$$
W_{(\varphi, \Delta H)}=\left[\begin{array}{cccc}
1 & 0 & 0 & 0 \\
0 & 1 & 0 & 0 \\
0 & 0 & 1 & -\Delta H \\
0 & 0 & 0 & 1
\end{array}\right] \cdot\left[\begin{array}{cccc}
\cos \varphi & \sin \varphi & 0 & 0 \\
-\sin \varphi & \cos \varphi & 0 & 0 \\
0 & 0 & 1 & 0 \\
0 & 0 & 0 & 1
\end{array}\right] .
$$

The azimuth axis and the pitch axis of the two-axis precision nonmagnetic turntable had a perpendicularity error $p$ because the two supports for the pitch swing portion were not strictly equal, and the perpendicularity error $p$ was the clip of the actual rotary axis in the in-plane direction and the $Y$-axis angle. The height difference $\Delta h$ and the bearing spacing $L$ determined the error value $p$ of the pitch axis. The relationship was as follows:

$$
p=\arctan \frac{\Delta h}{L}
$$

The pitching oscillating error was mainly composed of the axis error $\beta$ of the pitch axis, the accuracy $\Delta \theta_{2}$ of the pitch-axis rotation position, and the vertical accuracy $p$ of the pitch axis and the azimuth axis. The projection of the actual pitch axis in the XOY plane was at an angle $\beta$ to the $Y$ axis. The rotation matrix was an orthogonal matrix, which satisfied $R_{i}\left(\theta_{i}\right)^{-1}=R_{i}\left(\theta_{i}\right)^{T}=R_{i}\left(-\theta_{i}\right)$. According to the principle of homogeneous transformation of spatial coordinates, the pitch-axis error transfer matrix was obtained as follows:

$$
\begin{gathered}
I_{\beta}=\left[\begin{array}{cccc}
\cos \left(\theta_{2}+\Delta \theta_{2}\right) & 0 & -\sin \left(\theta_{2}+\Delta \theta_{2}\right) & 0 \\
0 & 1 & 0 & 0 \\
\sin \left(\theta_{2}+\Delta \theta_{2}\right) & 0 & \cos \left(\theta_{2}+\Delta \theta_{2}\right) & 0 \\
0 & 0 & 0 & 1
\end{array}\right] . \\
\cdot\left[\begin{array}{cccc}
1 & 0 & 0 & 0 \\
0 & \cos p & \sin p & 0 \\
0 & -\operatorname{sinp} & \cos p & 0 \\
0 & 0 & 0 & 1
\end{array}\right] \cdot\left[\begin{array}{cccc}
\cos \beta & \sin \beta & 0 & 0 \\
-\sin \beta & \cos \beta & 0 & 0 \\
0 & 0 & 1 & 0 \\
0 & 0 & 0 & 1
\end{array}\right] .
\end{gathered}
$$

The azimuth rotation error was mainly composed of the axis system error $\alpha$ of the azimuth axis and the positional accuracy $\Delta \theta_{1}$ of the pitch axis, as shown in Fig. 5.

The rotation error of the shaft was mainly the pure radial error and the inclination error $\alpha_{1}$ (the angle at which the actual position of the rotary shaft was rotated around the $X$ axis) and $\alpha_{2}$ (the angle at which the actual position of the rotary shaft was rotated around the $Y$ axis). That is, the angle between the projection of the actual azimuth axis on the $X O Z$ plane and the $Y$ axis was $\alpha_{1}$, and that between the projection and the $Y O Z$ plane was $\alpha_{2}$.

First, the azimuth axis was rotated counterclockwise $\alpha_{2}$ around the $Y$ axis, the angle was rotated clockwise $\alpha_{1}$ around the $X$ axis, and finally the azimuth axis $\theta+\Delta \theta_{1}$ was rotated counterclockwise. The outer frame completed the coordinate transformation in the case of shafting error and corner error. According to the principle of space coordinate transformation and Euler rotation transformation, the azimuth-axis error transfer matrix was obtained as follows: 
$O_{\alpha}=\left[\begin{array}{cccc}\cos \left(\theta_{1}+\Delta \theta_{1}\right) & \sin \left(\theta_{1}+\Delta \theta_{1}\right) & 0 & 0 \\ -\sin \left(\theta_{1}+\Delta \theta_{1}\right) & \cos \left(\theta_{1}+\Delta \theta_{1}\right) & 0 & 0 \\ 0 & 0 & 1 & 0 \\ 0 & 0 & 0 & 1\end{array}\right]$.
$\cdot\left[\begin{array}{cccc}\cos \alpha_{2} & 0 & -\sin \alpha_{2} & 0 \\ 0 & 1 & 0 & 0 \\ \sin \alpha_{2} & 0 & \cos \alpha_{2} & 0 \\ 0 & 0 & 0 & 1\end{array}\right] \cdot\left[\begin{array}{cccc}1 & 0 & 0 & 0 \\ 0 & \cos \alpha_{1} & -\sin \alpha_{1} & 0 \\ 0 & \sin \alpha_{1} & \cos \alpha_{1} & 0 \\ 0 & 0 & 0 & 1\end{array}\right]$.

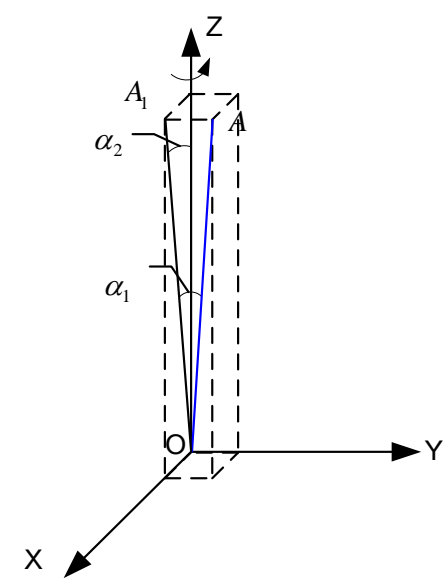

Fig. 5 Azimuth-axis error decomposition

An error inevitably occurred in the mounting base of the turntable. The error diagram is shown in Fig. 6.

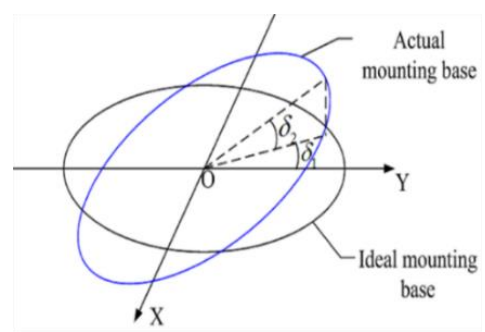

Fig. 6 Mounting base error

The actual mounting base was the ideal mounting base. It rotated $\delta_{1}$ counterclockwise around the $Z$ axis and then rotated $\delta_{2}$ clockwise around the $X$ axis. The error transformation matrix was as follows:

$$
\begin{aligned}
& A_{\delta}=\left[\begin{array}{cccc}
\cos \delta_{2} & 0 & \sin \delta_{2} & 0 \\
0 & 1 & 0 & 0 \\
-\sin \delta_{2} & 0 & \cos \delta_{2} & 0 \\
0 & 0 & 0 & 1
\end{array}\right] . \\
& {\left[\begin{array}{cccc}
\cos \delta_{1} & \sin \delta_{1} & 0 & 0 \\
-\sin \delta_{1} & \cos \delta_{1} & 0 & 0 \\
0 & 0 & 1 & 0 \\
0 & 0 & 0 & 1
\end{array}\right] .}
\end{aligned}
$$

The pointing error of the positioner could be calculated as follows:

$$
\sigma=\arccos \left(\frac{\vec{P}_{2} \cdot \vec{P}_{1}}{\left|\vec{P}_{2}\right| \times\left|\vec{P}_{1}\right|}\right) .
$$

\subsection{Influence of the turntable control on accuracy}

The pointing accuracy of the two-axis precision turntable was mainly the steady-state error, and the speed stability of the turntable was not high. Therefore, analyzing the positioning performance of the azimuth and pitch axes of the turntable in the step mode was important.

\subsubsection{Industrial computer control mode}

The following is an analysis of the steady-state error of the turntable to ensure that the control accuracy of the turntable was better than $0.01^{\prime}$. The azimuth axis was controlled to rotate at a step of $0.01^{\prime}$. According to Figure 7 , the steady-state angular error of the azimuth axis was $0.0014^{\prime}$, and the adjustment time of the azimuth axis was 0.7 s. According to Fig. 8, the positional accuracy of the pitch axis could reach $0.001^{\prime}$, and the adjustment time of the pitch axis could be up to $1.1 \mathrm{~s}$. The shortest time was $280 \mathrm{~ms}$.

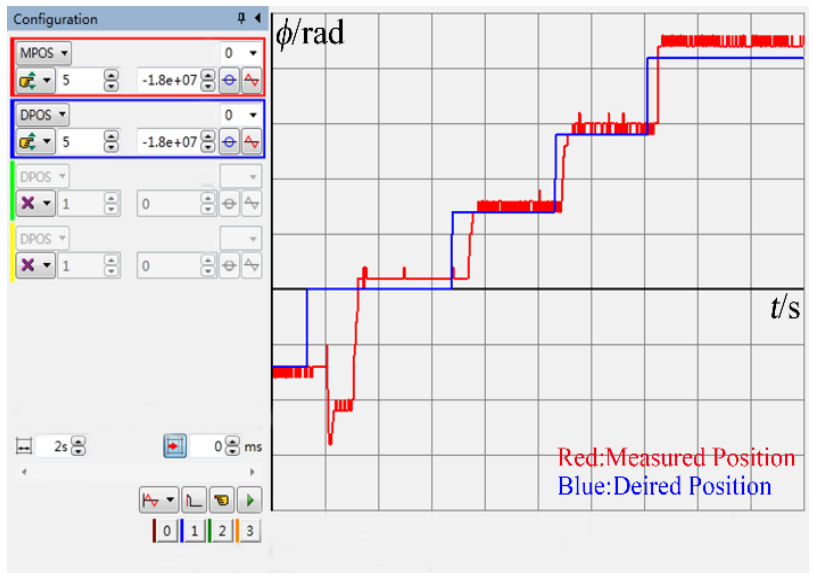

Fig. 7 Azimuth-axis steady-state error analysis

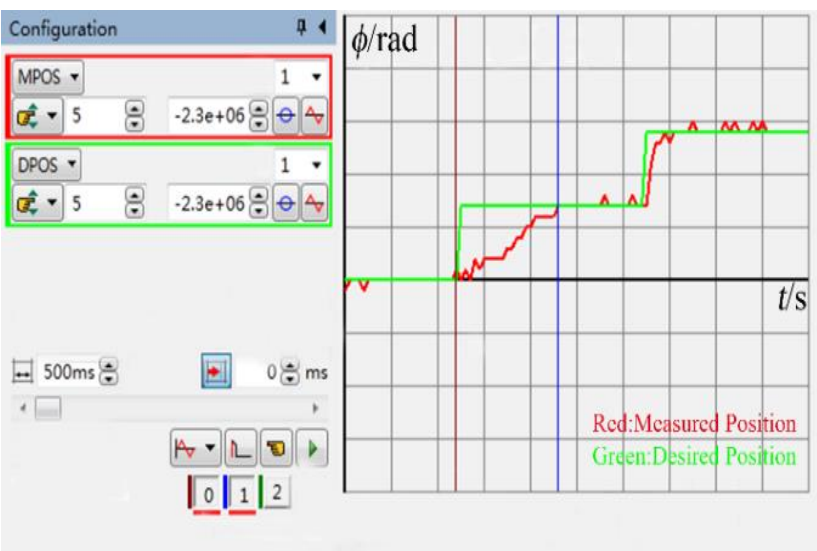

Fig. 8 Analysis of the steady-state error of pitch axis

By testing and analyzing the STEP control mode of the turntable control system, the step response curve was ideal. The steady-state following error of the azimuth and pitch axes was 0 , with no overshoot, but the system adjustment time was longer and the maximum. For a length of $1 \mathrm{~s}$, the adjustment time was inevitable. For a test turntable with a position accuracy of $0.01^{\prime}$, a certain adjustment time must be sacrificed to obtain a higher positional accuracy. 


\subsubsection{Joystick control mode}

The pulse hand wheel is a differential signal output with four signal outputs $\mathrm{A}+, \mathrm{A}-, \mathrm{B}+$ and $\mathrm{B}-$. In the pulse handle control mode, when the gear position switch is at the three speed levels of $\times 1, \times 10$, and $\times 100$, the rotational speeds of the azimuth axis and the pitch axis of the turntable are $0.01 \% \mathrm{~s}, 1^{\prime} / \mathrm{s}$, and $1^{\circ}$, respectively. Under the medium and high speeding gears, the response speed of the turntable is fast, there is no overshoot phenomenon, and the following error is small. In the $\times 1$ rate mode, the adjustment time of the turntable is long, there is no overshoot phenomenon, and there is slight noise in the steady state, as shown in Fig. 9.

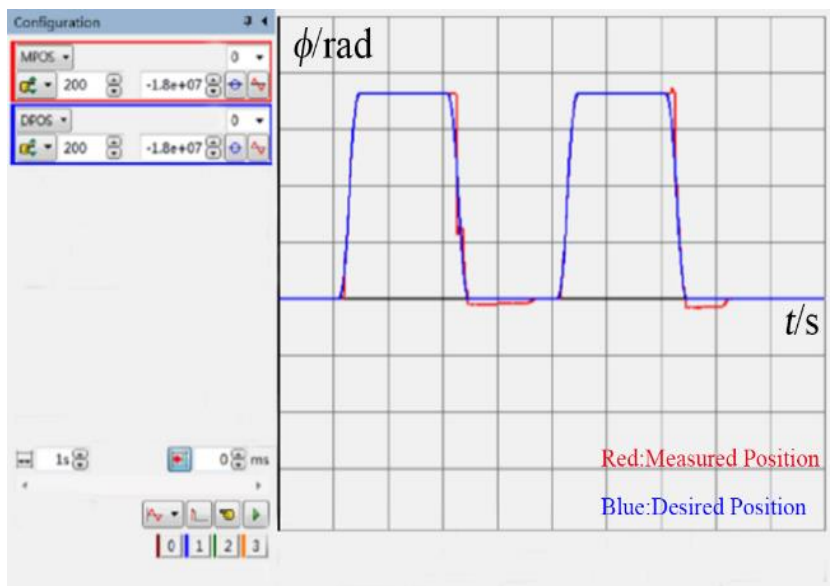

Fig. 9 Follow-up response in the pulse control mode

Based on the above results, the controllability of the two-axis precision turntable is very high, and the turntable uses an ultrasonic motor with a grating encoder. The minimum positional accuracy of the azimuth axis is 0.002 ', the positional accuracy of the pitch axis is 0.001 ', and the two-axis precision turntable can achieve a positioning accuracy better than 0.01 '. Moreover, the repeating positioning accuracy of the turntable is very good, which is also benefited from the absence of backhaul error in the direct drive mode of the ultrasonic motor. The two-axis precision non-magnetic turntable based on ultrasonic motor has high response speed and response time as short as $10 \mathrm{~ms}$. The control system does not have large overshoot and system oscillation, and is not sensitive to external vibration interference, which is also beneficial to ensure the accurate positioning performance of the two-axis turret [20].

\subsection{Summary of factors affecting the accuracy}

The factors affecting the accuracy of the turntable were grating encoder, mechanical body, and servo control parameters. The error caused by the grating encoder and the mechanical body could be reduced by the error compensation method, and the error caused by the servo control parameter could be reduced by introducing PID adjustment. How to reduce the error is explained in section 4 .

\section{PID motion control}

\subsection{Turntable equipment}

According to the specific working environment of the turntable and related design requirements, the preliminary design of the system composition of the two- axis precision turntable was carried out. The two-axis precision turntable was mainly composed of a mechanical structure body, a group of horizontal and pitch swing ultrasonic motors, two degrees-of-freedom grating encoders, motor drivers, controllers, PCs, and so on. The two-axis precision turntable system is shown in Fig. 10.

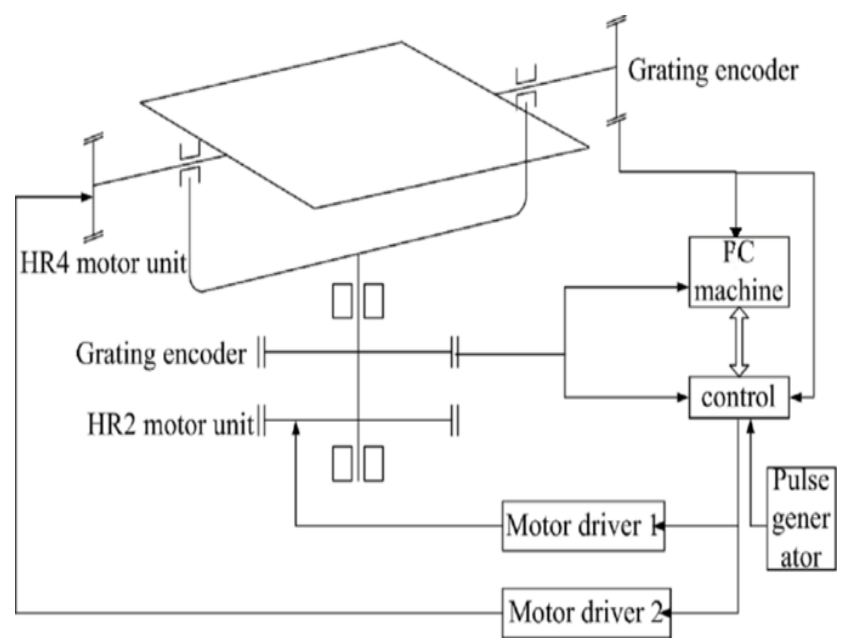

Fig. 10 Two-axis precision turntable system

The mechanical body is divided into an azimuth axis module and a pitch swing module, which makes the turntable easy to maintain and easy to ensure the installation accuracy. The main material of the two-axis precision turntable is aluminum alloy 7075 grade, which satisfies the rigidity of the turntable and does not make the magnetic field distortion. The shaft parts are made of 316L stainless steel, and the aluminum alloy 7075 is hard oxidized. Control system components include the Trio Euro-408 controller, HF2 driver, HF4 driver, 24V power supply, text display, joystick and more. The azimuth rotary shaft is made of ceramic bearing, and the alumina ring is bonded to the slewing ring by $3 \mathrm{M}$ acrylic tape. The HF2 ultrasonic motor unit directly drives the alumina ring by frictional coupling, and the pre-tightening force of the friction coupling is changed by the front and rear adjustable installation mode of the HF2 type ultrasonic motor. The attachable grating scale is mounted on the grating plate, and the azimuth axis grating read head is mounted on the adjustable bracket to facilitate the installation accuracy of the grating head. The azimuth rotary part is easy to assemble and disassemble, and only the round nut at the lower end of the rotary shaft needs to be unscrewed, and the azimuth shaft can be removed from the upper part.

The pitch swinging portion has four ceramic deep groove ball bearings supporting the pitch swinging body, and the ceramic bearing is embedded on the support seat, and the sliding friction with the rocking body is changed into rolling friction, and the precision is easily ensured. The gap adjusting screw and the gap adjusting block are used together to eliminate the gap between the pitching swinging body and the supporting seat. The gap adjusting block is made of PTFE, which has certain elasticity and good lubricating properties. The HF4 ultrasonic motor is installed symmetrically on the left and right sides, and the ultrasonic motor is easy to install and disassemble, and can achieve good maintainability. The ultrasonic motor presses the alumina sheet with a certain pre-tightening force, and the pre-tightening direction is a non-sensitive direction of the 
pitching pitch precision, and canceling each other does not increase the frictional resistance. The grating ruler is attached to the cylindrical surface of the pitching swing body, and the grating read head is mounted on the upper swing support plate to facilitate adjustment of the gap with the scale. The pitch swinging portion has the advantages of small friction torque, smooth motion, and high swing precision.

\subsection{PID motion control of the two-axis precision turntable}

\subsubsection{PID parameter tuning for rotation of the azimuth axis}

The positioning accuracy of the two-axis nonmagnetic precision turntable is very high, and it can reach the micrometer level, and the angular precision can reach 0.01 '. The following error of the two-axis non-magnetic precision turntable is very small, and the accumulator has a small accumulation error with time. It must wait for a long time to eliminate the steady-state error of the turntable, and the response speed of the non-magnetic turntable will be slower. Therefore, the integral term coefficient should be appropriately increased in the STEP positioning mode, which can increase the response speed of the turntable control system. It can be obtained through experiments that the proportional link $P=1$ is better, and Fig. $11, \mathrm{a}-\mathrm{c}$ is the step response motion curve when the integral link $I$ is different.

Fig. 11 a shows that when the integral term $I=0.05$, a motion command of $0.01^{\prime}$ rotation was performed on the azimuth axis of the turntable; the system step response could be measured as $t=3.2 \mathrm{~s}$ and no steady-state error was found. Fig. $11, \mathrm{~b}$ shows that when the integral term $I=0.06$, the system step response could be measured as $t=2.9 \mathrm{~s}$ and can be stable at the target position, no steady state error. Fig.11, c shows that when $I=0.08$, the system step response was $t$ $=0.3 \mathrm{~s}$. At this time, the system response speed greatly improved, the target position was quickly reached, and no overshoot and oscillation occurred.

The integral term $I$ continued to increase, and it was found that the turntable had a high probability of instability. and it is easily interfered by external vibration sources, and there is jitter or even divergence. At this time, the ultrasonic motor has a large noise. In the micro positioning mode, the control system is sensitive to errors, and the system introduces oscillation due to the vibration caused by the desktop and the noise introduced by the control system. Therefore, the integral link I should not take too much value on the premise of ensuring the response speed. Finally, the control parameters $P=1$ and $I=0.08$ are determined, and the adjustment effect is best.

\subsubsection{PID parameter tuning for rotation of the pitch axis}

The PID control parameter setting of the pitch axis of the two-axis non-magnetic precision turntable is similar to the azimuth axis tuning process. Firstly, the PID control parameter setting with the rotation angle of $1^{\circ}$ is performed, and the pitch axis of the turntable is rotated by $1.0^{\circ}$ by the controller. The controller displays the relationship between the actual position MPOS of the software turntable and the target position DPOS. The specific motion curve is shown in Fig. 12. It can be found that the following error in the motion of the turntable is very small, almost negligible, and there is no large instability such as overshoot and vibration. The PID control parameter at this time is $P=0.5 ; I=0.02$ the differential link can be omitted.

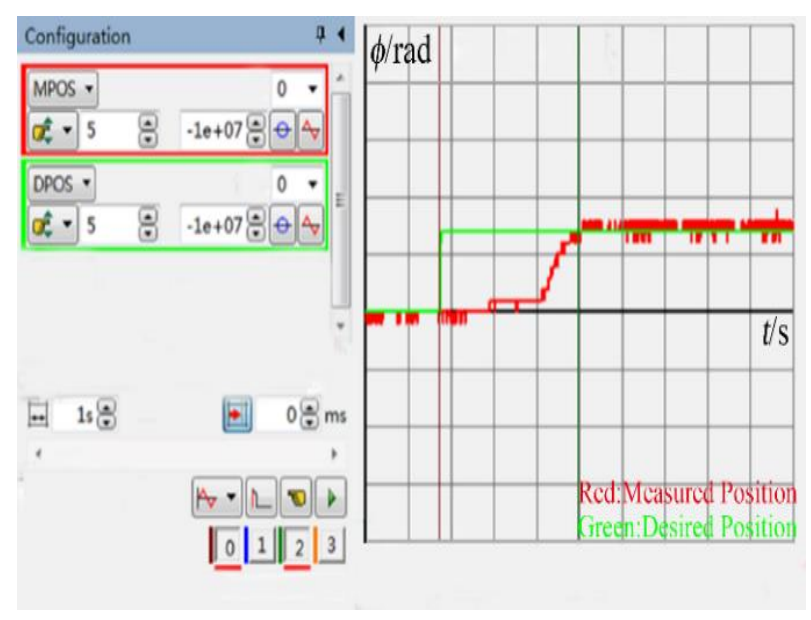

a) $P=1.0, I=0.05$

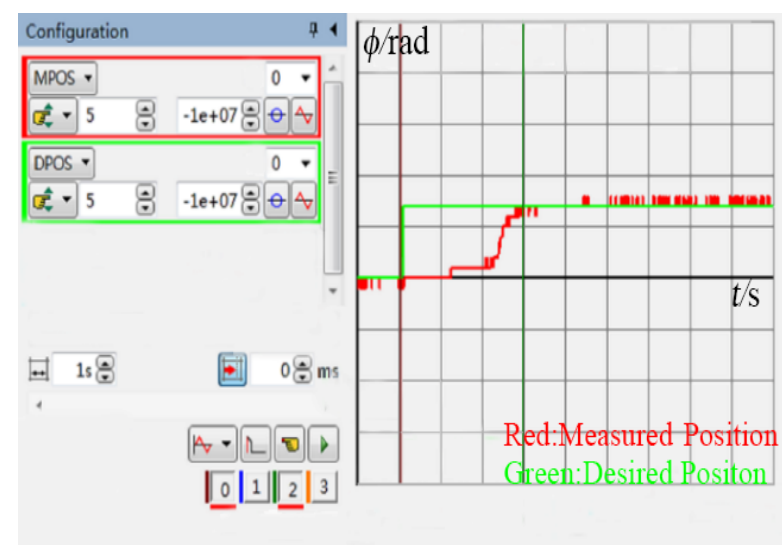

b) $P=1.0, I=0.06$

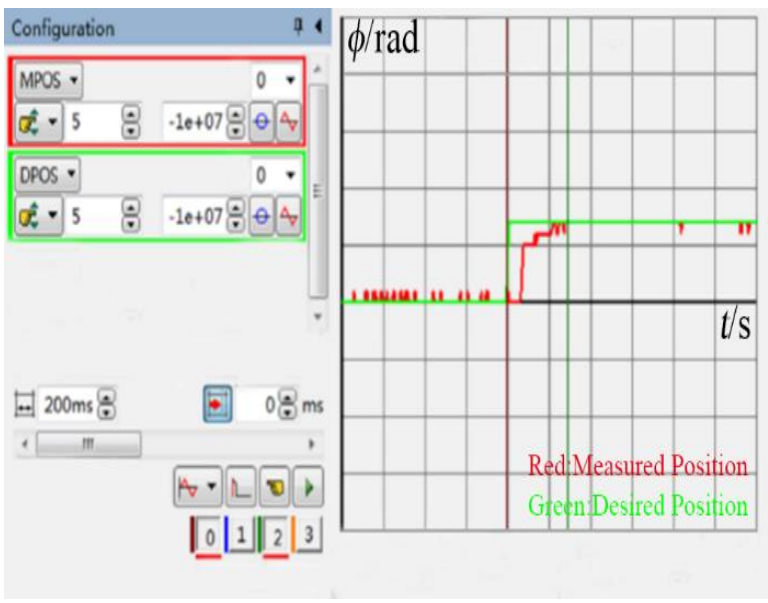

c) $P=1, I=0.08$

Fig. 11 Azimuth-axis PID parameter tuning

By setting the parameters of the two-axis precision turntable pitch axis at different angles, it was determined that the PID parameters at $1 \%$ rate were $P=0.5$ and $I=0.02$, as shown in Fig. 13; when the pitch-axis rotation speed was $1 \%$ s, $P=1.0$ and $I=0.1$. When the control turntable pitch axis worked in the STEP mode, the control system PID parameters were $P=2.0$ and $I=0.2$. With the aforementioned parameters, the pitch axis could run smoothly. 


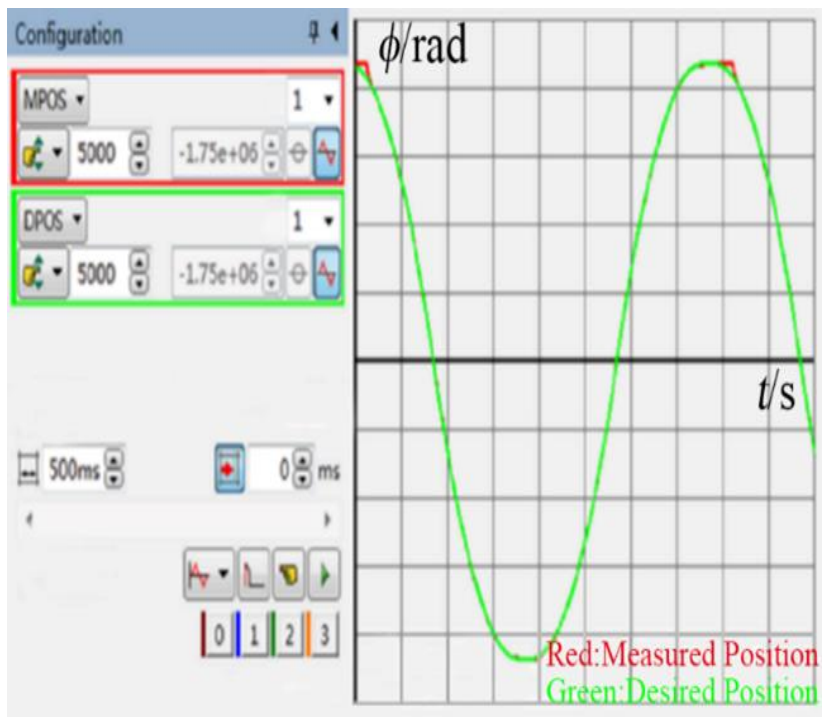

Fig. 12 Follow performance at $1^{\circ}$ pitch axis

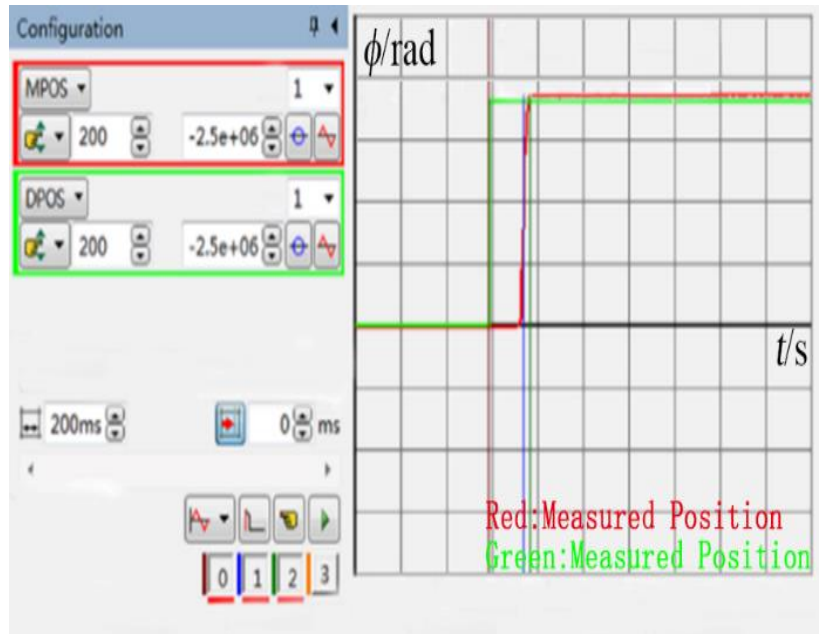

Fig. 13 Step response at $1^{\prime}$ speed of the pitch axis

\subsubsection{Analysis of positioning accuracy of the turntable}

The steady-state error of the azimuth axis and the pitch axis of the two-axis precision turntable was analyzed. The azimuth axis was controlled to rotate at a step of $0.01^{\prime}$, and the number of subdivision grids corresponding to one step was 7. According to Fig. 14, a and b, the steady-state angular error of the azimuth axis was 0.0014' and the adjustment of the azimuth axis to a steady state was obtained. The time was $0.7 \mathrm{~s}$.

Steady-state accuracy under PID control

Table 1

\begin{tabular}{|c|c|c|c|}
\hline & $\begin{array}{c}\text { Steady- } \\
\text { state error }\end{array}$ & Adjusting time & Overshoot \\
\hline $\begin{array}{c}\text { Azimuth } \\
\text { axis }\end{array}$ & $0.0015^{\prime}$ & $0.8 \mathrm{~s}$ & 0 \\
\hline Pitch axis & $0.0015^{\prime}$ & $1.0 \mathrm{~s}$ & 0 \\
\hline
\end{tabular}

By introducing PID parameters tuning to the turntable control system, as shown in Table 1, the step response curve obtained is ideal. The steady-state following error of the azimuth axis and the pitch axis is 0.0015 ', There is no overshoot. The system adjustment time is up to $1 \mathrm{~s}$. The longer adjustment time is unavoidable, but it can meet the requirements of the index. For test turrets with a position accuracy of 0.01 ', in order to achieve higher positional accuracy, a certain adjustment time must be sacrificed.

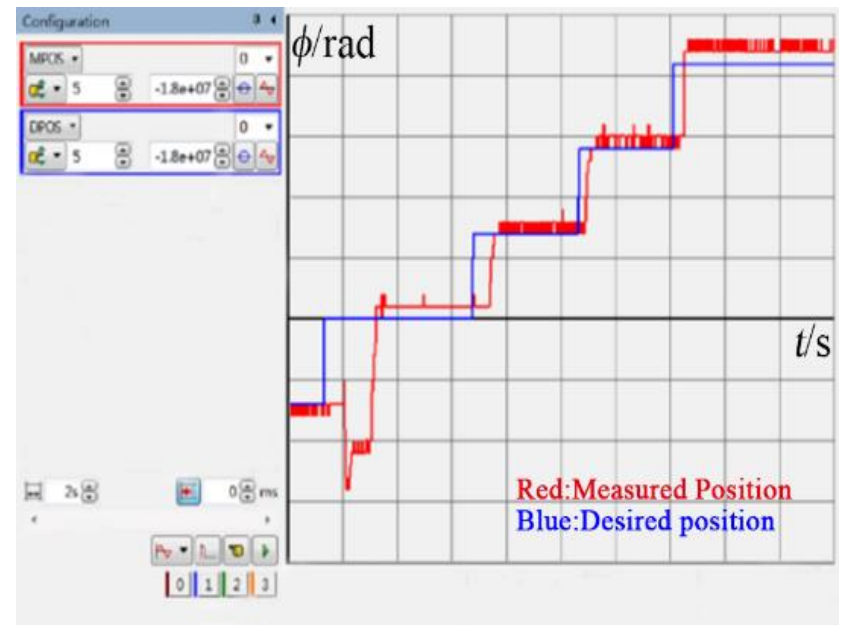

a) Azimuth axis steady-state error analysis

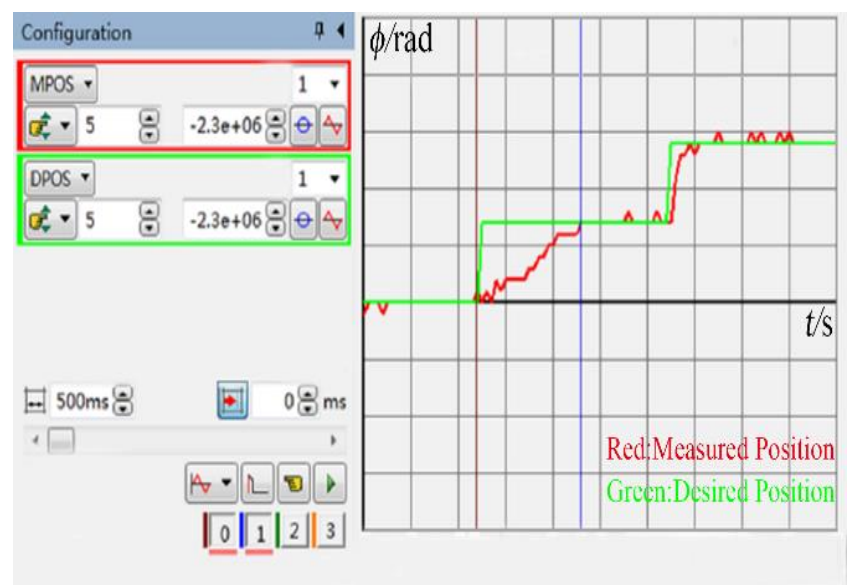

b) Steady state error analysis of pitch axis

Fig. 14 Control accuracy analysis of the two-axis turntable

\section{Conclusions}

In this paper, the accuracy analysis of the two-axis non-magnetic precision turntable is carried out. The accuracy mentioned here includes the static installation error of the non-magnetic turntable introduced by the mechanical body structure of the turntable and the steadystate positioning error introduced by the non-magnetic turntable control system. These two errors together cause the pointing error of the non-magnetic turntable.

By establishing a mathematical model for the influence of the installation static error on the overall pointing error of the turntable on the two-axis non-magnetic precision turntable, the specific influence degree of each error source of the non-magnetic precision turntable on the overall pointing error of the turntable can be calculated, and finally the pointing error of the non-magnetic turntable is found. The rotary position accuracy and the positioner installation error are the most sensitive, so the rotary table rotation error and the positioner installation error should be mainly controlled. The control system parameters of the two-axis precision turntable are adjusted to ensure that the turntable does not have excessive overshoot and oscillation during operation. The non-magnetic turntable has a fast response capability, and the steady-state errors of the 
azimuth axis and the pitch axis of the turntable are all less than 0.002 ', which can meet the requirements of the positioning accuracy index. On the whole, the accuracy of the two-axis precision non-magnetic turntable meets the design technical requirements, and the static parameters of the positioner can be tested and calibrated.

\section{Acknowledgments}

This study was funded by NSFC (Research on ultimate bearing capacity and parametric design for the grouted clamps strengthening the partially damaged structure of jacket pipes, grant no: 51879063; and Research on analysis and experiments of gripping and bearing mechanism for large-scale holding and lifting tools on ocean foundation piles, grant no: 51479043). The views expressed in this study are solely of authors.

\section{References}

1. Zhang, X. L.; Sun, B. Y.; Sun, J. W; et al. 2012 Pointing error analysis of high-precision twodimensional turntable, Journal of Changchun University of Technology, 33(4): 377-382. http://dx.doi.org/10.15923/j.cnki.cn22-1382/t.2012.04. 022.

2. Gao, G. H.; Wang, J. 2018.Structural design and modal analysis of a servo tracking turntable, Mechanical Design 35(S1): 151-154. http://dx.doi.org/10.13841/j.cnki.jxsj.2018.s1.035.

3. Du, Y. X.; Mi, Y.; Zhang, S. M. 2014. Application of variable structure control in high precision turntable system, Foreign Electronic Measurement Technology 33(09):68-71. http://dx.doi.org/10.19652/j.cnki.femt.2014.09.017.

4. Yao, X.; Sun, C.; Jin, Y.; et al. 2016. Development and error compensation of the high precision turntable, Chinese Journal of Scientific Instrument 37(5): 961-967. http://dx.doi.org/10.19650/j.cnki.cjsi.2016.05.001.

5. Zeng, M.; Wang, Z. S.; Su, B. K. 2006. Study on angular measuring system for the precision testing turntable, Journal of Harbin Institute of Technology 38(2): 167-139.

http://dx.doi.org/10.1677/jme.1.02008.

6. Xiao, S.Y.; Wang, T.; Jiang, X. Y.; et al. 2018. A spectrally tunable plasmonic photosensor with an ultrathin semiconductor region, Plasmonics 13(3). http://dx.doi.org/10.1007/ s11468-017-0586-1.

7. Wu, Y. 2015. Research status and development trend test table electronic production, (05): 240. http://dx.doi.org/10.16589/j.cnki.cn11-3571/tn.2015.05 .034 .

8. Zheng, J. 2019. Implementation of automatic test system for signal motor based on high-precision turntable, Micromotor 52 (07):82-84.

http://dx.doi.org/10.15934/j.cnki.micromotors.2019.07. 015.

9. Zhang, Q.; Wang, Q. J.; Li. Guo. 2016. Nonlinear modelling and predictive functional control of Hammerstein system with application to the turntable servo system, Mechanical Systems and Signal Processing 72-73: 383-394. http://dx.doi.org/10.1016/ j.ymssp.2015.09.011.

10. Chen, X. M.; Liu, C. J.; Du, B.L. 2019. Research on large-scale high-precision turntable control system, Electro-optical \& Control 26(05): 94-98. http://dx.doi.org/10.3969/j.issn.1671-637x.2019.05.01 8.

11. Wu, Y. K.; Huang, Y. F.; Liu, X. D. 2012. A compound internal model control method for high performance turntable servo system, Control and Decision Conference (CCDC), 24th (in Chinese). http://dx.doi.org/10.1109/ CCDC.2012.6244172.

12. Wen, C.; He, S.; Bu, C. et al. 2017 Cost-effective improvements of a rotating platform by integration of a high-accuracy inclinometer and encoders for attitude evaluation, Measurement Science \& Technology 28(1): 015901. http://dx.doi.org/10.1088/ 1361-6501/28/1/015901.

13. Chen Zhuzi; Chen Yu; Zhou Tieying. 2016. A hollow cylindrical linear nut-type ultrasonic motor, Mechanika 22(6): 546-552. https://dx.doi.org/10.5755/ j01.mech.22.6.13366.

14. Padgurskas, J.; Rukuiža, R.; Bansevičius, R.; et al. 2015. Impact of the tribological characteristics on the dynamics of the ultrasonic piezoelectric motor, Mechanika 21(1): 51-55. https://doi.org/10.5755/ j01.mech.21.1.10136.

15. Su, F. P.; Cui, W.2 018. Analysis and design of highprecision turntable, Sensor World 24 (07): 19-24. http://dx.doi.org/10.16204/ j.cnki.sw.2018.07.003.

16. Ye, C.; Cui, N. H. 2019. Research on sliding mode control of turntable servo system, Science and Technology (24):220. http://dx.doi.org/10.19392/j.cnki.1671-7341.20192419 6.

17. Lin, F. J; Xu, R. J.; et al. 2004.Two-axis motion control system using wavelet neural network for ultrasonic motor drives, IEE Proceedings - Electric Power Applications 151(5): 613-621. http://dx.doi.org/10.1049/ip-epa:20040685.

18. Jia, J. Y.; Chai, W.; Yu, D. L.; et al. 2016. Parameter identification and pointing accuracy analysis of azimuth pitching turntable, Chinese Journal of Scientific Instrument 37(7): 1500-1508. http://dx.doi.org/10.19650/ j.cnki.cjsi.2016.07.008.

19. Zhang, H. L.; Huang, K.; Zhao, Q. H.; et al. 2016. Improvement of coordinate calculation method for three-axis stabilized antenna of shipborne satellite communication earth station, Telecommunications Technology 56(2): 183-189.

http://dx.doi.org/10.3969/j.issn.1001-893x.2016.02.013.

20. Liu, Q.; Xi, J. 2011. Case-based parametric design system for test turntable, Expert Systems with Applications 38 (6): 6508-6516. http://dx.doi.org/10.1016/ j.eswa.2010.11.094. 
Z. Wang, X.T. Wang, T. Wang, B. Zhang, H.W. Ma

ACCURACY ANALYSIS AND MOTION CONTROL OF TWO-AXIS NONMAGNETIC TURNTABLE BASED ON ULTRASONIC MOTOR

\section{S u m m a r y}

In the calibration process of the positioner, it is necessary to test the rotation accuracy of the turntable than the accuracy of the target gyroscope to achieve the test and calibration of the positioner so as to obtain the accurate angular position information of each axis of the turntable. Therefore, this study used the nonmagnetic technology to explore the pointing accuracy and motion control of the turntable. Meanwhile, the influence of grating installation error on precision detection was analyzed. The influence of the steady-state error of turntable on the motion accuracy of the turntable was also analyzed. Finally, the influence of servo control parameters on the dynamic performance of turntable and the influence on steady-state error were analyzed. The test of the corner positioning accuracy of the turntable was carried out. The positioning accuracy and motion control parameters of the two-axis precision nonmagnetic turntable were obtained, and the PID adjustment was introduced to make the accuracy index of the nonmagnetic turntable meet the requirements. The turntable could realize the nonmagnetic working environment and achieve the high precision required by the index under the driving of the ultrasonic motor.

Keywords: control parameters, nonmagnetic environment, pointing accuracy, ultrasonic motor.

Received May 28, 2019

Accepted June 02, 2020 